\title{
One-stage surgical approach to coarctation of the aorta and ascending aortic aneurysm
}

\author{
Sabol F, Mistrikova L, Kolesar A, Luczy J, Toporcer T, Beres A \\ Clinic of Cardiac Surgery, East Slovak Institute of Cardiovascular Diseases, Kosice, Slovakia. \\ lmistrikova@vusch.sk
}

\begin{abstract}
Background: Aortic coarctation in adults is sometimes associated with concomitant cardiovascular pathologies which require intervention. The optimal operative approach for such patients remains unsettled. An extra-anatomic aortic bypass from the ascending aorta to the descending aorta via median sternotomy allows simultaneous performance of repair of complex aortic coarctation and concomitant cardiac operation. Case report: We present herein an adult female with coarctation of the aorta combined with an ascending aortic aneurysm associated with concomitant aortic valve regurgitation. We performed a single-stage operation which consisted of Bentall's procedure and extra-anatomic bypass from the ascending to the descending aorta. The patient's postoperative period was uneventful and twelve months after the surgery she is doing well.

The conclusions of our work resulted in one essential experience, namely that clinically serious, previously almost intractable conditions can be successfully resolved in unusual ways. Extra-anatomic reconstruction of aorta coarctation that for various reasons could not have been solved by resection of the affected aortic segment allowed us to achieve a structurally as well as functionally excellent outcome. Therefore, we recommend to consider this option in appropriate patients and/or incorporate it into the therapeutic armamentarium of cardiosurgical centers (Fig. 5, Ref. 11). Text in PDF www.elis.sk.

Key words: coarctation, aorta, bypass, congenital malformation.
\end{abstract}

Coarctation of the aorta is a common congenital defect which, unless primarily detected and surgically corrected in childhood, may remain undiagnosed until adulthood. In most patients it is then discovered during examination of systemic hypertension (1). Moreover, coarctation is commonly associated with concomitant cardiac congenital or acquired pathology that may require surgical intervention. In addition, $5 \%$ to $30 \%$ of patients with previous coarctation repair have recoarctation and require reintervention (2).

Aortic coarctation is a serious pathology which requires surgical or endovascular treatment. About $50 \%$ of cases of uncorrected isolated aortic coarctation are lost up to the age of 10 ; only $10 \%$ may reach the age of 50 . The most common reason for death from untreated aortic coarctation is the rupture of aortic aneurysm or side branches with a rate of $23 \%$ (3).

The management of aortic coarctation associated with other cardiac pathology in adults appears to be a great surgical challenge. Insufficient clinical data due to the small number of these patients on the one hand and a large variety of complicating factors on the other are the reasons why there is no consensus on the optimal approach. Debates exist not only as to which lesion should be corrected first but also in regard to the type and timing of the

Clinic of Cardiac Surgery, East Slovak Institute of Cardiovascular Disease, Kosice, Slovakia

Address for correspondence: F. Sabol, MD, PhD, Clinic of Cardiac Surgery, East Slovak Institute of Cardiovascular Disease, Ondavska 8, SK04011 Kosice, Slovakia.

Phone: +421.55.7891011 procedure. Surgery can be one- or two-staged (4). An alternative surgical approach is an anatomic left-sided short aortic bypass from the arcus aorta to the descending aorta via median sternotomy, which allows a simultaneous repair of a complex aortic coarctation and concomitant cardiac operation (5).

We present herein an adult female patient with coarctation of the aorta combined with an ascending aortic aneurysm and associated aortic valve regurgitation. We performed a single-stage operation which consisted of Bentall's procedure and extra-anatomic ascending-to- descending aortic bypass. The patient's postoperative period was uneventful and twelve months after the surgery she is doing well as can be seen on CT reconstructive angiography at follow up (Fig. 1).

\section{Case report}

A 28-year old female was referred to our hospital because of recurrent fever poorly reacting to antibiotic treatment and newly diagnosed bicuspid aortic valve with severe aortic insufficiency. The patient had a medical history of hypertension which started at the age of 18 and was poorly controlled with a combination of antihypertensive agents.

The patient's problems started six months ago after recovering from unspecified viral infection. Since then she has suffered from fatigue, exertional dyspnea and recurring fever in spite of repeated antibiotic therapy. The patient was classified as functional class NYHA II. Upon admission she was hemodynamically stable with normal temperature; her arterial blood pressure 


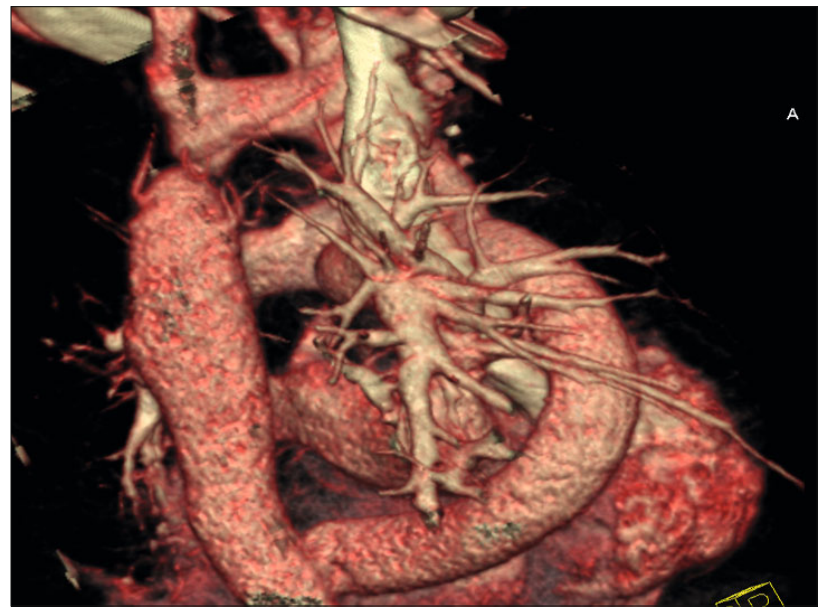

Fig. 1. CT angiography after 12 months (follow up). Aortic coarctation and the bypass clearly visible.

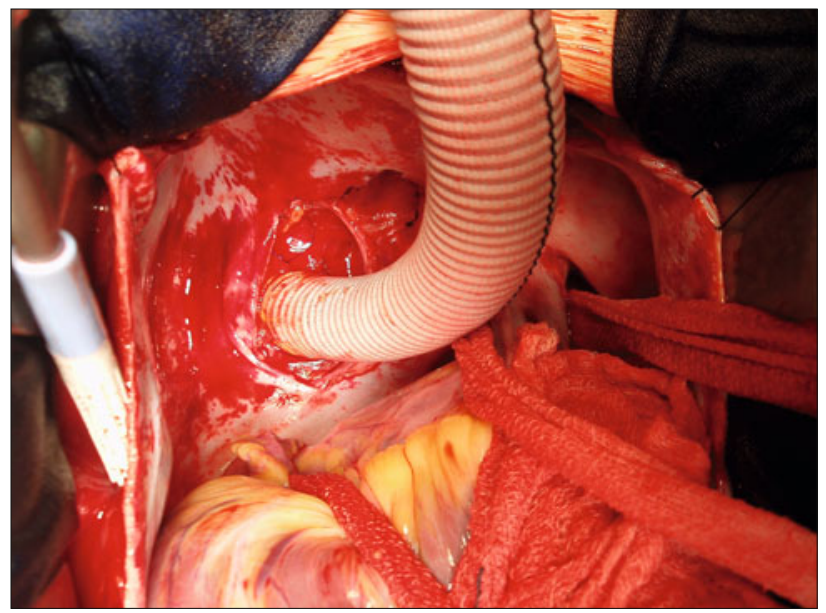

Fig. 2. Distal anastomosis - tubular graft to a descending aorta (visible after verticalization of the heart).

was 160/60 mmHg in both upper extremities, and 80/50 mmHg in both lower extremities. Physical examination revealed grade 3/6 diastolic murmur heard best at the cardiac apex, while radial pulses were normal. However, the femoral pulses were weak. Chest X-ray illustrated rib notching and cardiomegaly. Cardiac cathetrization showed normal coronary arteries and severe aortic coarctation with gradient $88 \mathrm{mmHg}$ measured invasively and severe aortic valve insufficiency. Transesophageal echocardiography revealed a bicuspid aortic valve with severe aortic regurgitation, aortic root dilatation of $50 \mathrm{~mm}$, ascending aorta of $44 \mathrm{~mm}$, and left ventricular ejection fraction of $60 \%$. Furthermore, it showed a membranous narrowing of the descending aorta approximately $20 \mathrm{~mm}$ distally from the origin of the left subclavian artery with a minimum diameter of the stenotic region less than $5 \mathrm{~mm}$. The membrane gave rise to a strong suspicion of endocarditis because of the billowing structure of $6 \mathrm{~mm}$ present on the distal side of the membrane. Magnetic resonance angiography and CT scan confirmed previous findings. Repeated hemocultures had negative results.

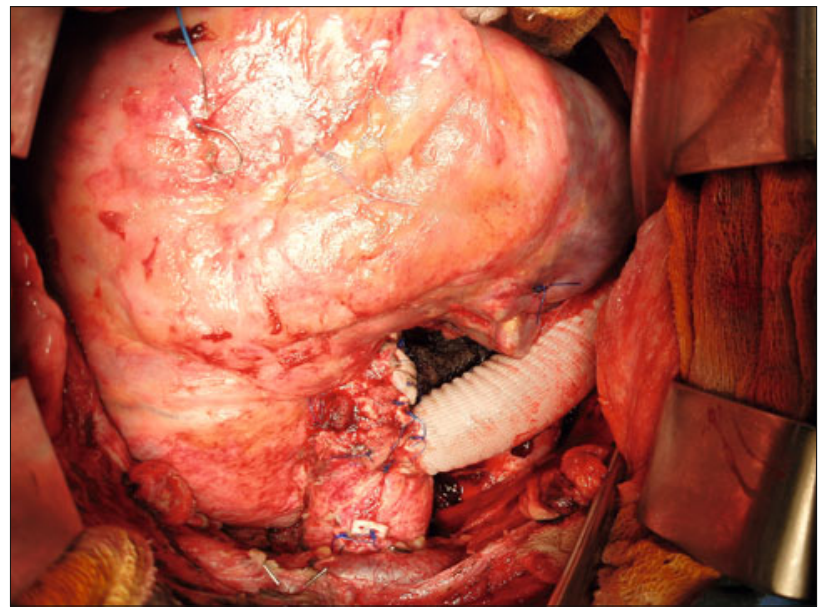

Fig. 3. Complete reconstruction.

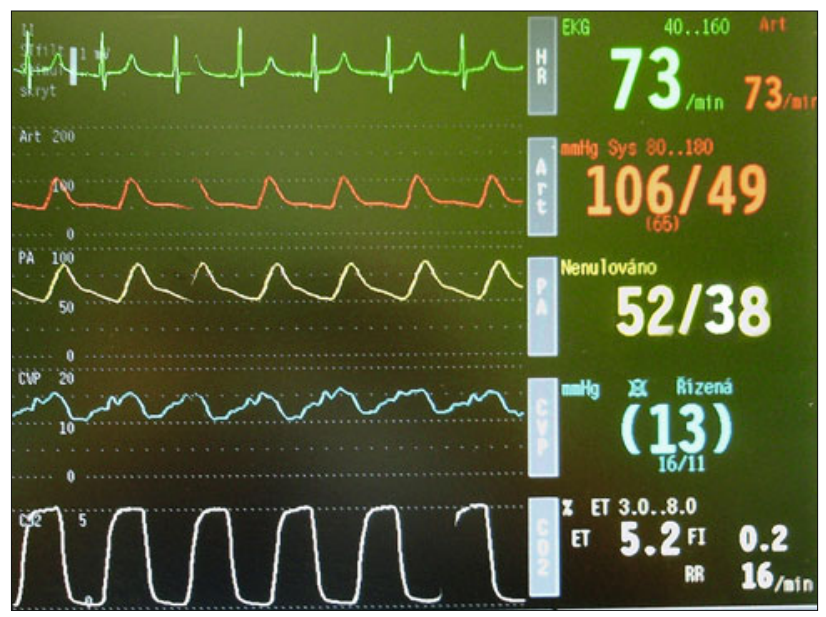

Fig. 4. Invasive arterial blood pressure monitoring before the surgery. The significant difference between the blood pressure in a. radialis $(106 / 49 \mathrm{mmHg}$ ) and a. femoralis $(52 / 38 \mathrm{mmHg})$.

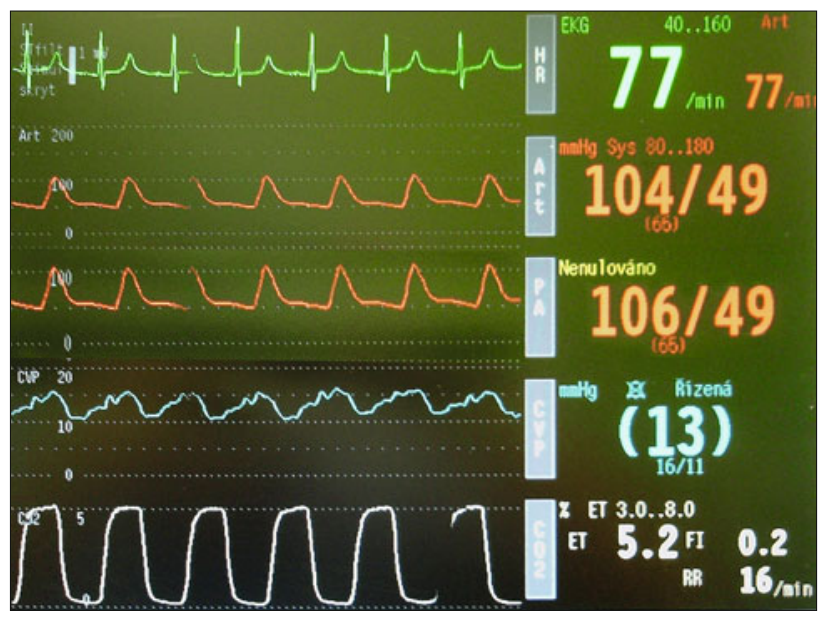

Fig. 5. Invasive arterial blood pressure monitoring after the complete reconstruction. Equalizing the blood pressures measured in a. radialis $(104 / 49 \mathrm{mmHg})$ and a.femoralis $(106 / 49 \mathrm{mmHg})$. 
Surgery was performed via median sternotomy using single venous cannulation while the temperature of the patient was lowered to $28{ }^{\circ} \mathrm{C}$. After instituting the cardio-pulmonary bypass (CPB), the ascending aorta was cross-clamped, cardioplegic solution was administered to coronary ostia, and the descending aorta was mobilized in posterior mediastinum. The aortic wall was very fragile. Using a site-clamp we performed distal anastomosis (Fig. 2) with a tubular graft (size 18) to the descending aorta. We continued with an inspection of the aortic valve which was bicuspid with a prolapse of the anterior fused cusp. Furthermore, we noticed more perforations and healed vegetation placed on the ventricular side of the non-coronary cusp. We evaluated that the valve was not suitable for repair. A sample was taken in order to perform bacteriological examination. Considering the findings we decided for valve replacement and performed Bentall's procedure using a mechanical conduit (size 23/25). After finishing the distal anastomosis of a conduit to the distal part of the ascending aorta we released the cross-clamp. The graft from the descending aorta was then placed between the inferior vena cava and right inferior pulmonary vein, around the left atrium and above superior vena cava to the ascending aorta. After reconstructing the bypass and Bentall's procedure (Fig. 3) we monitored the equalizing of blood pressures measured from a. radialis and a. femoralis (Figs 4 and 5). Because of prolonged diffuse bleeding we decided to postpone the chest closure, which was performed on the following day. The postoperative period was uneventful; the patient was transferred to a ward on the 6th postoperative day, and discharged from the hospital on the 14th postoperative day. Bacteriological examination of the valve was negative, and therefore prolonged antibiotic treatment was not recommended.

At the follow up, six months after the surgery, the patient was asymptomatic and CT scanning did not show any pathologic findings.

\section{Discussion}

Coarctation of the descending thoracic aorta generally manifests itself in childhood. (5) However, if coarctation is left untreated, $25 \%$ of such patients will die by the end of their 2nd decade of life, $49 \%$ by the end of their 3rd decade and approximately $90 \%$ by the age of 50 years. Causes of death in patients with aortic coarctation are aortic rupture, aortic dissection, congestive heart failure, bacterial endocarditis, and intracranial hemorrhage (6).

Aortic coarctation may represent a single surgical entity or may occur in a more complex form with congenital or acquired intracardiac pathology, where additional surgical intervention is mandatory (1).

Since the first surgery for coarctation repair in 1944 (7), there have been debates concerning the optimal surgical approach, timing of surgery, and the whole management of the patient. The surgery of aortic coarctation still represents a great challenge for the surgeon since there are no existing guidelines. Correction of complex forms can be performed as a one-stage repair of both lesions through median sternotomy or as a two-stage repair through median sternotomy and lateral thoracotomy. Complex forms have been surgically treated using anatomic repair and extra-anatomic bypass grafting. Anatomic repair my be complicated by the necessity of extensive mobilization of the aorta, control of collateral blood vessels, possible parenchymal lung injury, damage to the recurrent laryngeal or phrenic nerves, chylothorax and spinal cord ischemia $(2,8)$. Paraplegia and the risk of spinal cord injury remain the most frightening complications of the aortic surgery. Various extra-anatomic bypass techniques have been introduced throughout the years for the management of complex or isolated forms of coarctation with or without establishment of a cardiopulmonary bypass $(4,8)$. Non-surgical interventional procedures are possible for native or recurrent coarctation. However, these procedures are not feasible or desirable in all patients, and longterm outcome data are not available $(2,9)$.

Vijayanagar et al (10) were the first to describe aortic valve replacement and concomitant ascending-to-descending aortic bypass through the posterior pericardium with graft placement around the left margin of the heart, completely performed via median sternotomy. Since then, there have been several modifications of this technique introduced by several authors. For example, Powell et al placed the graft around the right margin of the heart (11).

Paraplegia is rarely encountered when using the extra-anatomic correction of coarctation. An aortic side-clamp does not block the blood flow to the branches of the aorta. This allows continuation of blood flow to the intercostal arteries and, in turn, reduces the risk of paraplegia (2).

There are only few long-term reports concerning bypass grafting for coarctation. Potential late complications include graft narrowing or graft kinking, infection, anastomotic dehiscence or development of a false aneurysm. Yilmaz et al (5) suggest that the risk of kinking of the aortic bypass might be lowered by using an anatomic left-sided aortic bypass.

If coarctation with its concomitant cardiac pathologies requires surgical intervention, a single-stage approach for the treatment of coarctation and cardiac defect using cardiopulmonary bypass is a safe surgical alternative. At this time coarctation is treated by extra-anatomic ascending-to-descending bypass. This combined approach avoids potential complications of the anatomic repair and allows concomitant procedure through the same incision. The graft is placed between the inferior vena cava and right inferior pulmonary vein in posterior location in order to avoid compression of the right atrium. At the same time, in case of inevitable resternotomy, the prosthesis is protected from potential surgical injury (2).

\section{Conclusion}

In spite of the growing importance of endovascular techniques, surgery still plays an important role in the management of adult patient with coarctation of the aorta. The surgical management of patients with complex coarctation or re-coarctation with or without associated cardiac disorders must be individualized. An extra-anatomic coarctation bypass appears to be a safe and flexible method that is particularly useful in adult patients when simultaneous intracardiac repair is necessary. 


\section{3-596}

\section{References}

1. Maron B, Humphries J, Rowe RD, Meliits ED. Prognosis of surgically corrected coarctation of the aorta. A 20-year postoperative appraisal. Circulation 1973; 47: 119-126.

2. Connolly HM, Schaff HV, Izhar U, Dearani JA, Warnes CA, Orszulak TA. Posterior Pericardial Ascending-to-Descending Aortic Bypass: An Alternative Surgical Approach for a Complex Coarctation of the Aorta. Circulation 2001; 104 (Suppl 1): I-133-I-137.

3. Erkut B, Kaygin MA, Dag O, Ates A. Single Stage Operation with Two Different Incisions in a Patient with Ascending Aortic Aneurysm and Aortic Coarctation. Firat Med J 2010; (15) 4: 204-206.

4. Koletsis E, Ekonomidis S, Panagopoulos N, Tsaousis G, Crockett J, Panagiotou M. Two stage hybrid approach for complex aortic coarctation repair. J Cardiothor Surg 2009; 4: 10.

5. Yilmaz M, Polat B, Saba D. Single-stage repair of adult aortic coarctation and concomitant cardiovascular pathologies: a new alternative surgical approach. J Cardiothor Surg 2006; 1: 18.
6. Hiller N, Verstanding A, Simanovsky N. Coarctation of the aorta associated with aneurysm of the left subclavian artery. Br J Radiol 2004; 77 (916): 335-337.

7. Crafoord C, Nylin G. Congenital coarctation of the aorta and its surgical treatment. J Thorac Surg 1945; 14: 347-361.

8. Grinda J, Mace L, Dervanian P et al. Bypass graft for complex forms of isthmic aortic coarctation in adults. Ann Thorac Surg 1995; 60: 1299 1302.

9. Weber H, Cyran S. Initial results and clinical follow up after ballon angioplasty for native coarctation. Am J Cardiol 1999; 84: 113-116.

10. Vijayanagar R, Natarajan P, Eckstein PF, Bognolo DA, Toole JC. Aortic valvular insufficiency and postductal aortic coarctation in the adult. Combined surgical management through median sternotomy: a new surgical approach. J Thorac Cardiovasc Surg 1980; 79 (2): 266-268.

11. Powell W, Adams P, Cooley D. Repair of coarctation of the aorta with intracardiac repair. Tex Heart Inst J 1983; 10: 409-413.

Received March 13, 2013. Accepted April 20, 2014. 